Instituto Internacional de Investigación y Desarrollo Tecnológico Educativo INDTEC, C.A.

DOI: https://doi.org/10.29394/scientific.issn.2542-2987.2016.1.2.15.254-273

OAI-PMH: http://www.indteca.com/ojs/index.php/Revista Scientific/oai

\title{
Integración del Triángulo Escolar en las Actividades Sociopedagógicas y Culturales de la U.E.E. "Hilarión Alberto Viloria"
}

\author{
Autor: Wilmer José Pérez Rojas \\ Universidad Nacional Experimental "Rafael María Baralt", UNERMB \\ elprof 7@hotmail.com \\ Zulia, Venezuela
}

\section{Resumen}

El objetivo de esta investigación fue desarrollar un plan de acción para la integración del triángulo escolar a las actividades socio pedagógica y cultural de la U. E. E. Hilarión Alberto Viloria. Para lograr este objetivo se abordó la metodología propuesta por Pérez (2000), en sus cuatro fases: diagnóstico, planificación, ejecución y evaluación. Se concluyó que los docentes constituyen el elemento fundamental para realizar las transformaciones sociales en los cambios paradigmáticos de la educación donde se le brinde una serie de oportunidades para producir cambios y transformaciones no solo en estrategias organizativas, sino en valores inmersos en las prácticas pedagógicas. Con respecto a la planificación, tomando en cuenta los resultados arrojados en el diagnóstico, se hizo necesario planificar planes de acción fundamentados en la participación de los actores sociales. Se debe propiciar la inclusión de valores de sentido de pertenencia y sustentabilidad entre los miembros de la escuela y la comunidad, favoreciendo en los actores sociales un cambio de actitud que propicien culturas del buen ciudadano hacia los estilos de vida sustentables.

Palabras clave: integración escuela-comunidad; motivación comunitaria; el docente como ente transformador de cambios. 


\section{School Integration Partner Triangle in Educational and Cultural Activities of U.E.E. "Hilarion Alberto Viloria"}




\section{Introducción}

En el contexto histórico actual, la educación afronta situaciones que la desafían a buscar caminos que hagan más efectiva su acción en los educandos. Uno de estos retos es la incorporación de la familia a la escuela; se considera que es necesaria una acción conjunta familia - escuela que dinamice la formación integral del educando. Al hablar de acción conjunta, se pretende involucrar a los padres y representantes en cada aspecto de la educación y desarrollo de sus hijos, desde el nacimiento hasta la edad adulta, puesto que es la familia el primer centro educativo donde la persona adquiere valores y hábitos, que serán reforzados por la escuela.

A la escuela le corresponde, además de educar al niño(a), extender su acción a la familia, desplegando estrategias y acciones concretas que permitan a los padres y representantes adquirir herramientas y conocimientos que les ayuden a educar a sus hijos(as). Padres, representantes y docentes, son los agentes para lograr el ideal educativo que persigue la escuela y, cuyo fin último, es la búsqueda de la realización personal del educando

Uno de los objetivos que se plantea la Educación Integral en Venezuela es mejorar la integración entre la escuela y la familia, logrando un desarrollo integral de los niños(as) para que sean personas estables y equilibradas, que se vinculen a la sociedad en una forma creativa. La formación integral de los niños(as) no es una tarea que depende sólo del maestro, también es responsabilidad de la familia incorporarse a las actividades pautadas dentro del aula para así aprender diversas estrategias y trabajarlas en conjunto con los niños(as) en el hogar. La familia es el grupo humano primario más importante en la vida del hombre, según lo especifican distintos autores, entre ellos Ojeda (2008); "es considerada como la institución más estable de la historia de la humanidad". El hombre vive en familia, aquella en la que nace, y posteriormente, la que el mismo crea. Es innegable que cada hombre o mujer, al unirse como pareja, aporta a la familia recién creada su manera de pensar, 
sus valores y actitudes; trasmite luego a sus hijos los modos de actuar con los objetos, la forma de relación con las personas, las normas de comportamiento social que reflejan mucho de lo que ellos mismos en su temprana niñez y durante toda la vida aprendieron e hicieron suyo en sus respectivas familias, para así crear un ciclo que vuelve a repetirse.

Es fundamental que los padres y/o representantes comprendan su papel protagónico y puedan estar informados de las actividades realizadas en la jornada diaria dentro del aula de clases. En tal sentido, la decisión será más fácil en la medida en que los padres tengan un conocimiento real de las habilidades y necesidades de sus hijos(as), sepan qué les ofrece la alternativa escolar e identifiquen sus propias inquietudes, necesidades, habilidades, capacidad de compromiso y participación en el proceso educativo. Según Bitar (2007):

Educar con calidad requiere que usted y su familia se comprometan con la escuela y que padres, madres y apoderados(as) estén atentos a lo que aprendan sus hijos(as) y en estrecha alianza con el profesor para apoyar su trabajo desde el hogar

En el contexto de la Educación Primaria, es frecuente que la participación de los padres y representantes sea poco activa, debido, entre otras razones, a limitaciones de tiempo, disposición, y a la situación socioeconómica que se vive actualmente. Esta situación se contrapone a la necesidad de que los padres y representantes colaboren con los maestros en el aula y adquieran el conocimiento de las estrategias que se plantean en la jornada de trabajo con los niños(as), para luego ser reforzadas en el hogar.

Los padres son los responsables de educar al niño(a), inculcándole valores y sus propias enseñanzas; los docentes se encargan de reforzar esta educación sobre la base de las potencialidades de los pequeños alineándose a los reglamentos del Ministerio del Poder Popular para la Educación, que 
establecen una integración entre ambos actores: padres - docentes para mediar las experiencias significativas al educando.

\section{Teoría y Conceptos}

\subsection{Contextualización del Problema}

En la actualidad, se observa que los padres y/o representantes están desligados de las actividades escolares de sus hijos, por lo que no se interesan en preguntar o participar en la jornada de trabajo de los mismos. En el momento de la entrada, los padres y/o representantes dejan a sus hijos en la puerta y no se preocupan en pasar a verificar si está o no el docente, tampoco notifican cuando los niños se sienten mal o están enfermos. A la hora de la salida, algunos niños los retira el vecino, o los padres y representantes llegan hasta la puerta del plantel a retirar al representado, sin pasar a preguntarle al docente ¿cómo se portó?, ¿cómo va?, ¿cuáles actividades realizaron hoy?

En Venezuela la educación tiene la responsabilidad de cumplir con la transformación de los ciudadanos a través de una educación integral, ya que esta le permite desenvolverse en su entorno socio-cultural y ser capaz de resolver problemas de la vida cotidiana, por eso la educación integral forma parte de las necesidades de los niños y niñas para el logro de sus objetivos como medio de integración social. Existe una corriente educativa que viene imponiéndose a nivel mundial en la de Educación Primaria, y no es otra que la Integración Escolar; es decir, la incorporación de educandos con necesidades en escolaridad regular, pudiéndose interpretar como estrategia para promover la integración social del individuo con necesidades en la Educación Primaria para enriquecer su calidad de vida.

El propósito de esta investigación es detectar de qué forma se puede controlar la situación de los padres y representantes acerca la gestión pedagógica, que ocurre dentro y fuera de la institución, en este caso en la Unidad Educativa Estatal "Hilarión Alberto Viloria", de tal manera que se 
sientan motivados en cuanto a la toma de conciencia, y que propicien la participación, el dialogo, la justicia, la autonomía, y la democracia; para legitimar normas sustentadas en valores, que conduzcan a una convivencia escolar armónica. Esto permitirá formar personas aptas para incorporarse a otros niveles educativos y en otros espacios en la vida, con sentido pertenencia dignidad, fraternidad y justicia.

En los actuales momentos, es bien sabido de que la familia por sí sola, como institución ya no resuelve por completo las necesidades de la persona, y la escuela por sí sola tampoco logra solventar las múltiples vertientes del proceso socializador; por lo tanto el análisis de las continuidades 0 discontinuidades entre familia y escuela se convierte en un elemento clave para comprender mejor los determinantes ambientales del desarrollo. Si la familia es el agente educador por excelencia y delega parte de esta responsabilidad en las Instituciones educativas, éstos deben caminar juntos en el proceso educativo formativo. El proceso educativo supone una simbiosis de elementos (docentes, familia, comunidad) en fluida integración para así lograr el desarrollo del potencial de sus estudiantes.

La comunidad educativa, la asociación de padres y representantes, conjuntamente con los docentes y educandos, constituyen la verdadera base del proceso educativo consciente, basado en la realidad y centrado en valores. La asociación de padres, conjuntamente con las comunidades educativas, es una fuente inagotable de riqueza integrativa y constituyen un poderoso eslabón cogestor en la escuela y la familia.

La integración facilita el contacto personal entre los padres y los docentes, canaliza las sugerencias e iniciativas de padres, docentes, y alumnos. La interacción entre la familia y la escuela constituye el modo central para la educación de los hijos que son los mismos alumnos. Si quieren sobrevivir como instituciones, familia y escuela han de integrarse; esto se logra abriendo las puertas de la institución a las familias y la comunidad, invitando a 
charlas, talleres, vivencias, convivencias, fiestas, juegos, reuniones y más reuniones, hasta que tomemos conciencia de la importancia, y necesidad de esta integración y se convierta en una actividad consciente y habitual, en una rutina agradable e indispensable.

Tomando en consideración lo planteado anteriormente, se establecen las siguientes interrogantes: ¿Qué situación en el contexto escolar, generan determinados comportamiento en los padres y representantes, de tal manera que la gestión pedagógica de los docentes se integren con la comunidad?, ¿Cuáles son las causas que generan la desintegración Escuela-Comunidad?, ¿Qué factores inciden en el funcionamiento de la integración Escuela Comunidad?,¿Qué estrategias administrativas se utilizan en la actualidad para promover la integración familia, escuela y comunidad en la Educación Primaria?, ¿Consideran los actores del hecho educativo que la Integración Escuela Comunidad es un factor indispensable para optimizar el proceso enseñanza aprendizaje, de los educandos?

Ante esta problemática, los actores que hacemos vida activa en la institución, consideramos que el problema planteado motiva a abordar esta investigación, con el propósito de diseñar estrategias que permitan la integración de padres, representantes y comunidad con la Unidad Educativa Estatal "Hilarión Alberto Viloria" con el propósito de mejorar la convivencia escolar y permitir la participación de todos en las actividades académicas programadas durante el año escolar.

\subsection{Objetivo General}

Diseñar estrategias que promuevan la integración escuela, familia y comunidad, factor indispensable en la optimización del proceso enseñanza aprendizaje en los educandos de Primaria de la Unidad Educativa Estatal "Hilarión Alberto Viloria". 


\section{Fundamentación teórica del problema socio pedagógico}

En este compendio, se realiza la revisión de bibliografías, en las cuales se pueden mencionar autores, que ya han realizado trabajos de investigación sobre el tema, que sirven de ayuda, ya que ofrecen material que sirve de apoyo al desarrollo de la presente investigación.

Castillo, M. (2007), realizo un trabajo de investigación para la Universidad Pedagógica Experimental Libertador - Instituto Pedagógico de Maturín, titulada "La Integración Escuela Comunidad en función de lograr una mejor actuación y formación del alumno. Caso de Estudio: E.B "Luisa Teresa Sosa. Santa Elena de las Piñas Maturín-Monagas", concluyó: Existen limitaciones que no permiten que haya una acción comunicativa, por tal razón se recomienda actividades motivadoras para alcanzar una buena actuación y formación del alumnado en su aprendizaje.

Morocoima, B. (2008), en su trabajo de grado realizado para la Universidad Pedagógica Experimental Libertador - Instituto Pedagógico de Maturín, titulado: "Propuestas dirigidas a Incorporar a los padres y/o representantes a participar en el proceso de enseñanza y aprendizaje de los alumnos de la II Etapa de la Escuela Básica Santa Inés. Municipio Caripe Estado Monagas". Concluyo: es necesario formar una iniciativa de preparación de los padres que atienden a las características particulares de la comunidad a la cual sirve.

\subsection{Escuela.}

El término escuela tiene varios significados, entre ellos:

- Lugar o edificio donde se enseña y se aprende.

- Institución que tiene por objeto la educación.

- Conjunto de profesores y alumnos de una misma enseñanza

- Diversas concepciones metódicas. 
- Corriente del pensamiento, del estilo o agrupamiento de los seguidores de un maestro.

- Aquello que alecciona o da experiencia.

En nuestro país, se denomina escuela, al recinto donde conviven docentes y estudiantes, dentro del cual se produce el acto educativo a través del proceso enseñanza- aprendizaje, ordenados por una estructura rígida y formal, que viene dada por lo estipulado en el Currículo Nacional Bolivariano.

\subsection{Comunidad.}

Es el grupo o conjunto de personas que comparten elementos culturales y sociales comunes, tales como lenguaje, historia, ubicación geográfica, actividades; además, formar parte de ella, los organismos, entes y personas que tengan vinculación con el desarrollo de la comunidad en general.

\subsection{Integración Escuela-Comunidad.}

En el sistema educativo nacional existe una larga tradición en las escuelas de entablar proyectos con la comunidad desde diferentes perspectivas. Es así que se establecieron diferentes modalidades de vinculación que han sido importantes y han contribuido a generar formas creativas que colaboraron tanto en el quehacer educativo de la escuela como en mejoras en la vida de la comunidad.

Una de las modalidades que podemos reconocer es aquella que piensa a la escuela y a su cultura como un elemento externo a la comunidad. La función de la escuela es socializar según los valores de la sociedad. Desde esta perspectiva, la escuela es la transmisora de una única cultura. La escuela se coloca por encima de los problemas sociales de la comunidad que la rodea y a la vez la integra. En esta modalidad la escuela se propone generar algunas actividades de extensión hacia la comunidad sin la participación de la misma. 
En este tipo de vínculo al no tomar en cuenta las condiciones sociales de los alumnos a la hora de aprender no siempre la relación redunda en un beneficio educativo.

En este vínculo, se inscribe con una larga e importante trayectoria el Programa Organización Bolivariana de Familia (OBF), del Ministerio del Poder Popular para la Educación. Esta propuesta se basa en la idea de aprendizaje - servicio que es una metodología pedagógica que desarrolla proyectos solidarios o de participación comunitaria. Se plantea que existe un alto impacto en el aprendizaje de niños y jóvenes a partir del vínculo solidario. Es una modalidad que refuerza uno de los aspectos principales de la educación de los sujetos que hace referencia a la educación en valores para lograr conformar una mejor ciudadanía.

Se ha desarrollado en esta breve síntesis sólo algunas de las modalidades posibles de vinculación entre la escuela y su comunidad sabiendo que existen múltiples experiencias que exceden al recorte realizado. Es necesario recuperar los conceptos centrales de cada modalidad para poder construir una propuesta que posibilite avanzar en la tarea educativa en el difícil contexto social, cultural, económico y político por el que atraviesa nuestro país.

\subsection{Motivación Comunitaria}

La motivación está constituida por todos los factores capaces de provocar, mantener y dirigir la conducta hacia un objetivo. Por ello, el docente y la escuela en general están llamados a focalizar con el fin de lograr su objetivo. La motivación se refiere a las condiciones o estados que activan o dan energía al organismo, que conducen a una conducta orientada hacia determinados objetivos. También se considera la motivación como "...el proceso para despertar la acción, sostener la actividad en progreso y regular el patrón de actividad". 
La coyuntura que actualmente atraviesa el país está signada por una dominación económica, legitimada a través de la noción de progreso, y con expresiones concretas en el ámbito cultural. La globalización, a través de los medios de comunicación, se expande sin fronteras, evidenciando la necesidad de considerar con sumo cuidado los términos de intercambio entre la dimensión local y global. La investigación propone al estado una intervención activa para construir la participación ciudadana, desde su definición hasta su promoción y ejecución, y donde la escuela funcione como uno de los ejes centrales. Adicionalmente al contexto o situación, debemos considerar los deseos y necesidades, que de manera positiva impulsan a las comunidades a reaccionar, a relacionarse con el aprendizaje.

\subsection{El docente como actor de la integración social.}

El principal rol que debe cumplir el docente como actor de integración es iniciar y motivar a los demás miembros de la comunidad de aprendizaje, monitorear el intercambio dialógica de los grupos e intervenir cuando lo considere necesario; diseñar actividades alternativas para el logro de los objetivos; evaluar el proceso de aprendizaje, grupal e individual; realizar retroalimentación de las diferentes actividades e introducir modificaciones oportunas.

En la relación docente - comunidad, es importante destacar que cada comunidad tiene singularidad con diferencias que son propias. Cada individuo que forme parte de una comunidad posee una necesidad de identificación cultural y de pertenencia, que es necesario satisfacer, a través de la educación.

Por lo tanto, la relación docente - educando - comunidad debe tener entre sus principales características de aprendizaje, el brindar situaciones lo más concretas posibles y vivenciales con su entorno natural, cultural y social. Dentro de la comunidad está la familia, que pertenece a una cultura y, por lo 
tanto, en la medida en que se favorezca esa relación, se afirman también los lazos de valoración y estima de ella.

\section{Fundamentos Pedagógicos}

\subsection{Constructivismo}

El constructivismo es un cuerpo de teorías que tienen en común la idea de que las personas, tanto individual como colectivamente, construyen sus ideas sobre su medio físico, social o cultural. De esa concepción de construir el pensamiento surge el término que ampara a todos. Puede denominarse como teoría constructivista, por tanto, toda aquella que entiende que el conocimiento es el resultado de un proceso de construcción o reconstrucción de la realidad que tiene su origen en la interacción entre las personas y el mundo.

Por tanto, la idea central reside en que la elaboración del conocimiento constituye una modelización más que una descripción de la realidad. Al respecto, el Currículo Básico Nacional (1997) expresa:

Si se considera una visión pedagógica de la educación, puede afirmarse que sus fines o funciones inherentes lo constituye la adaptación, la socialización y la endoculturización, así como también la creación y transformación cultural y la integración personal como parte de la misión renovadora, innovadora y transformadora que la educación debe cumplir.

Por su parte, Belén Sanjuán propone la educación integral como la única capaz de asegurar un mejor futuro para el país. Esto lo dice en base a sus propias experiencias durante un movimiento renovador llamado "Escuela Nueva", el cual no permaneció debido a sus principios filosóficos y éticos, que estaban orientados hacia la formación de un ser crítico y capaz contribuir con el desarrollo del país. 
Según Belén Sanjuán (1999):

La educación integral es, un modelo pedagógico, cuyo objetivo principal es desarrollar la total personalidad de hombres y mujeres, de manera que éstos estén preparados para desenvolverse ante cualquier situación de la vida. Es por esto que esta educación involucra todas las áreas del saber, no intenta depositar conocimientos que no puedan ser entendidos y que no sean de provecho para los estudiantes, sino que pretende enseñar a aprender para que así sigan aprendiendo a lo largo de la vida, y además enaltece en gran medida el trabajo y los valores propios de la comunidad.

\section{Metodología}

La investigación se enmarca en los postulados de Pérez (2000), bajo el enfoque cualitativo con el tipo de investigación acción participativa (IAP), pues en el estudio de la problemática el objetivo general fue desarrollar un plan de acción para la integración del triángulo escolar a las actividades socio pedagógica y cultural de la U. E. E. Hilarión Alberto Viloria. Para ello, el investigador tomó en cuenta las cuatro fases señaladas a continuación:

Diagnóstico: Es el proceso donde se pueden detectar las problemáticas y los recursos disponibles de la comunidad para luego poder efectuar acciones en función del objetivo propuesto.

Planificación: Se organizan los talleres dirigidos a la familia-escuelacomunidad, a fin de concienciarlos en cuanto al grado de responsabilidad que tienen para con el proceso de enseñanza aprendizaje de sus representados.

Ejecución: Es la fase donde se desarrolla el plan de acción para la integración de los padres y representantes a las actividades socio pedagógicas culturales de la U. E. E. Hilarión Alberto Viloria.

Evaluación: Es la fase en la cual se hace una revisión de las actividades realizadas a través de un cuadro comparativo resaltando los logros alcanzados. 


\section{Análisis de Datos}

\subsection{Evaluación de los resultados.}

La evaluación es un componente ineludible de todo proyecto educativo que aspira a ser válido y eficaz. Esta evaluación ha de plantearse, en todo caso, con una finalidad esencialmente formativa, lo que implica que ha de llevarse a cabo con el propósito firme de utilizarla para mejorar los resultados, para optimizar el proceso de ejecución, si fuera preciso, para reconsiderar los objetivos propuestos.

Debe, pues, constituir un elemento potenciador del replanteamiento constante todo el proyecto en sus diferentes fases. La evaluación de un proyecto puede entenderse como "un proceso sistemático, diseñado intencional o técnicamente, de recogida de información valiosa y fiable orientado a valorar la calidad y los logros del mismo, como base para la posterior toma de decisiones de mejora, tanto de dicho proyecto, como del personal implicado, de modo indirecto, del cuerpo social en que se encuentra inmerso" (Pérez, 2000).

En consecuencia, el análisis de los resultados, logrados o no, del proyecto debe hacerse teniendo en cuenta los objetivos planteados, los cuales deben conducir a la elaboración de las conclusiones y propuestas; estas últimas se orientarán al reforzamiento de los aciertos y a la modificación, si acaso, de los aspectos menos satisfactorios. En todo caso, ofrecerá una visión global del proyecto, así como el detalle pertinente de los aspectos específicos de los diferentes sectores, áreas y ámbitos concretos.

\subsection{Proceso de Evaluación}

A través del diagnóstico realizado en la U. E. E. Hilarión Alberto Viloria., se pudieron detectar las necesidades más pertinentes para poder diseñar estrategias que promuevan la integración escuela, familia y comunidad, factor indispensable en la optimización del proceso enseñanza aprendizaje en los 
educandos de Primaria de la Unidad Educativa Estatal "Hilarión Alberto Viloria".

Por consiguiente, se pudo constatar resultados concretos de las metodologías empleadas en los talleres, los cuales fueron en función de generar una nueva visión de los padres y representantes en cuanto a su participación en las actividades culturales de la institución donde sus hijos y representados asisten, con la intención de promover la cultura del buen ciudadano y estilos de vida sustentable.

Aunado a lo anterior, con la ejecución de los talleres programados, se logró despertar en los padres y representantes una perspectiva mayor de su rol en la gestión educativa, específicamente en las áreas pedagógica y cultural, donde estos mostraron gran receptividad a las temáticas desarrolladas por los ponentes, asimismo se mostraron participativos, interviniendo durante el desenlace del taller, discerniendo ideas y opiniones, al mismo tiempo aportando alternativas de soluciones a los planteamientos propuestos.

En referencia a la aplicación del plan de acción para la integración de los padres y representantes a las actividades culturales, se determina que el mismo permitió propiciar cambios significativos que contribuyeron a la innovación de los conocimientos, por consiguiente, se obtuvo que los padres y representantes concibieron el plan propuesto, lo cual se evidenció por medio del dinamismo mostrado en la actividad, estableciéndose nuevos vínculos entre ellos mismos, docentes, directivos, y estudiantes.

Finalmente, la evaluación está compuesta por diversas acciones u operaciones relativas a la determinación de los objetivos, del objeto, la obtención y procesamiento de información, la elaboración de un juicio evaluativo, la retro-información y toma de decisiones derivadas del mismo, su aplicación y valoración de resultados. 


\section{Conclusiones}

La escuela es el motor de cualquier cambio en la sociedad del futuro. El sistema escolar debe formar ciudadanos conscientes, cultivados con una visión clara de la historia que les haga conocer en cada momento las consecuencias de hechos sociales remotos y menos remotos. Con el dominio oral y escrito más completo posible de su lengua materna, el estudiante actual será el ciudadano del mañana. Crítico, tolerante y exigente con sus conciudadanos teniendo de esta manera un estilo de vida sustentable.

En este sentido, se consideró importante diseñar estrategias que promuevan la integración escuela, familia y comunidad, factor indispensable en la optimización del proceso enseñanza aprendizaje en los educandos de Primaria de la Unidad Educativa Estatal "Hilarión Alberto Viloria"; ya que, la formación integral de los niños y niñas tienen que ir al unísono con esta triada antes señalada. La escuela es un medio privilegiado para ello, es indispensable para la formación de la masa crítica ciudadana, que en todo momento pueda atraer un cambio de los que no toleran las prácticas sociales o políticas dominantes.

Desde esta perspectiva, los docentes constituyen el elemento fundamental para realizar las transformaciones sociales en los cambios paradigmáticos de la educación donde se le brinde una serie de oportunidades para producir cambios y transformaciones no solo en estrategias organizativas, sino en valores inmersos en las prácticas pedagógicas. Con respecto a la planificación, tomando en cuenta los resultados arrojados en el diagnóstico, se hizo necesario planificar planes de acción fundamentados en la participación de los actores sociales.

Los cambios de la sociedad actual son rápidos y profundos, los sujetos no están preparados para adaptarse a ellos en los diversos niveles: biológico, psicológico y social. La complejidad, cada vez mayor, que la caracteriza, 
demanda una nueva visión educadora de la familia y la escuela, lo que exige su compromiso para trabajar unidas en un proyecto común.

Favorecer una educación para estilos de vida sustentable, a través de la cultura del buen ciudadano, desde el ámbito familiar, es fundamental para que el educando aprenda a ejercitar la participación, la cooperación, el diálogo y la toma decisiones consensuadas para compartir los conocimientos y la vida, de forma que luego sea capaz de transferir estos aprendizajes a otros contextos sociales.

En este sentido, un importante objetivo es que la escuela-familiacomunidad tome conciencia de la necesidad de su participación en ámbitos sociales más amplios, que influyen en sus prácticas educativas en el hogar. Este planteamiento, fue sentido por la comunidad del sector de San Rafael de El Tigre y la U. E. E "Hilarión Alberto Viloria".

Para lograrlo, es preciso ayudarla a descubrir la importancia del trabajo en colaboración, aceptando que existen objetivos que son comunes en los que son necesarios unir los esfuerzos para su consecución. De esta manera, el presente proyecto permitió trazarse como objetivo un plan de acción que fomente el sentido de pertenencia en los padres y representantes en pro de la integración en la ejecución de los proyectos educativos de la U. E. E. Hilarión Alberto Viloria.

Para alcanzar este objetivo se abordó la metodología bajo la modalidad de proyecto social según Pérez (2000), a través de cuatro fases: diagnóstico, planificación, ejecución y evaluación. El diagnóstico facilitó conocer las necesidades o problemáticas de la comunidad y la institución educativa, utilizando diversas técnicas como la entrevista, observación, lluvia de ideas, grupo nominal, planes de acción.

En cuanto a la planificación, se tomó en consideración los resultados arrojados en el diagnóstico participativo, lo cual conllevó a planificar tres 
talleres propiciando una convivencia entre la escuela-comunidad. Estos planes se ejecutaron sin contratiempos lográndose con esto los objetivos planteados.

Por último, se realizó la evaluación de cada una de las actividades planificadas resaltando sus logros. Quedando demostrado que fomentar una cultura ciudadana para el desarrollo de un estilo de vida sustentable en la escuela-familia-comunidad es clave fundamental para la optimización de la educación de calidad. La acción conjunta y continuada a través de actividades durante el proyecto promovió el conocimiento mutuo que rompió con las visiones sesgadas y parciales de las personas.

\section{Recomendaciones}

De acuerdo a los resultados obtenidos en la investigación, se hacen las siguientes recomendaciones:

La puesta en marcha de una campaña que incentive el deseo de suscitar cambios en la escuela-comunidad.

Incentivar en los docentes el deseo de involucrarse en el trabajo comunitario e involucrar a los padres y representantes como una familia que buscan un bien común del educando.

Crear instrumentos a nivel institucional para promover una convivencia con un estilo de vida sustentable.

Fomentar relaciones de apoyo brindando acceso a los miembros de la comunidad inmediata para servir a las familias que necesitan para una mejor calidad de vida sustentable.

Crear un clima de respeto y confianza, que propicie lazos de fraternidad entre escuela-familia-comunidad.

Fortalecimiento de la organización social en torno al desarrollo de procesos sostenibles de convivencia que permitan intensificar las acciones, especialmente el derecho a la paz y la sana convivencia. 


\section{Referencias}

Bitar (2007). "La sensibilización de las comunidades". 3era edición. Editorial Romus. Barcelona.

Castillo (2007). "Formación en valores". 2da edición. Volumen 3. Tomo I. Madrid: Editorial Taurus.

Morocoima (2008). "Teorías para la nueva sociedad”. Santander: Fundación Marcelino Botín.

Ojeda (2008). "El árbol del problema como técnica". 2da edición. Editorial McGraw-Hill. Madrid.

Pérez (2000). "Elaboración de proyectos sociales de la investigación científica". Madrid: Editorial Tecnos.

Sanjuán (1999). "El proceso de formación de valores de convivencia y su marco referencial”. 2da edición. Editorial Alfa. Caracas. 


\author{
Wilmer José Pérez Rojas \\ e-mail: elprof 7@hotmail.com
}

Nació en Pueblo Nuevo Estado Zulia, el 30/04/1983.

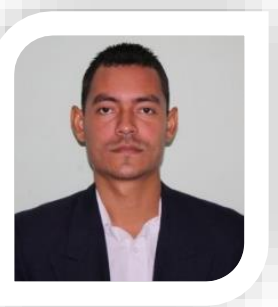

Estudiante de la Universidad Nacional Experimental "RAFAEL MARÍA BARALT" en el DOCTORADO EN EDUCACIÓN. Estudios de postgrado: Universidad "Valle de Momboy". Título obtenido: ESPECIALISTA EN PLANIFICACIÓN EDUCACIONAL y en la Universidad Nororiental Gran "Mariscal de Ayacucho", título obtenido MAGISTER EN GERENCIA EDUCATIVA. Estudio de Pregrado: U.N.E.R.M.B. Titulo Obtenido: LICENCIADO EN EDUCACIÓN MENCIÓN INTEGRAL. Ha participado en cursos, talleres y simposios sobre "Fundamentos y principios en la evaluación de los aprendizajes" "Introducción a la Computadora". "Formación de Equipo de alto desempeño". "Ingles Instrumental" "Taller de Expresión Oral y Escrita". "Participación de los Programas Fortalecimiento en la Familia". "Higiene y Seguridad Industrial" Proceso de Sensibilización Crecimiento y Liderazgo" Recapacitación y Actualización en el Nuevo Diseño curricular "Educación para los Valores Humanos". Transformación y Formación Educativa. "Liderazgo Educativo" "Calidad Educativa" "Sectorización e Investigación Social en Salud" "Proyecto Pedagógico de Aula" "Estrategias Didácticas para un aprendizaje Significativo" "Estrategias para la Enseñanza de la lengua oral y la Lengua Escrita". Maestro de Aula en la U.E.E. "Hilarión Alberto Viloria" San Rafael de El Tigre, Municipio Baralt Estado Zulia desde 2003 hasta la actualidad. Profesor en la UBV desde 2009 hasta 2014, Profesor universitario en el IUTEHPI desde el 2015 hasta la actualidad. 